Short communication

\title{
Evaluation of vaccinal effectiveness of preparations containing membrane antigens of Leishmania (L.) amazonensis in experimental cutaneous leishmaniasis model
}

\author{
João G. Ribeiro ${ }^{\mathrm{a}, \mathrm{b}}$, Amália S. Ferreira ${ }^{\mathrm{a}}$, Sharon R.A. Macedo ${ }^{\mathrm{a}}$, Norton R.D.L.P. Rossi ${ }^{\mathrm{a}}$, \\ Mayara C.P. da Silva ${ }^{c}$, Rosane N.M. Guerra ${ }^{c}$, Neuza B. de Barros ${ }^{\mathrm{a}}$, Roberto Nicolete, ${ }^{\mathrm{a}, \mathrm{d}, *}$

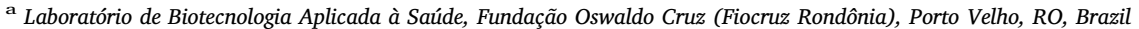 \\ b Instituto Federal do Amazonas (IFAM), Campus Humaitá, Humaitá-AM, Brazil \\ c Laboratory of Immunophysiology, Department of Pathology, Center for Biological and Health Sciences, Federal University of Maranhão (UFMA), São Luís, MA, Brazil \\ ${ }^{\mathrm{d}}$ Fundação Oswaldo Cruz (Fiocruz Ceará), Fortaleza, CE, Brazil
}

\section{A R T I C L E I N F O}

\section{Keywords:}

L. amazonensis

Membrane fraction

Vaccines

PLGA micro/nanoparticles

Protection

\begin{abstract}
A B S T R A C T
American tegumentary leishmaniasis (ATL) is considered a neglected disease, for which an effective vaccine or an efficient diagnosis is not yet available and whose chemotherapeutic arsenal is threatened by the emergence of resistance by etiological agents such as Leishmania amazonensis. ATL is endemic in poor countries and has a high incidence in Brazil. Vaccines developed from native parasite fractions have led to the identification of defined antigenic subunits and the development of vaccine adjuvant technology. The purpose of the present study was to develop and compare preparations based on membrane antigens from L. amazonensis, as a biotechnological prototype for the immunoprophylaxis of the disease in a murine experimental model. For this purpose, batches of biodegradable polymeric micro/nanoparticles were produced, characterized and compared with other parasite's antigens in solution. All preparations containing membrane antigens presented low toxicity on murine macrophages. The in vivo evaluation of immunization efficacy was performed against a challenge with $L$. amazonensis, along with an evaluation of the immune response profile generated in BALB/C mice. The animals were followed for sample processing and quantification of serum-specific cytokines, nitrites and antibodies. The sera of animals immunized with the non-encapsulated antigen formulations showed higher intensities of nitrites and total IgGs. This approach evidenced the importance of the biological studies involving the immune response of the host against the parasite being interconnected and related to the subfractionation of its proteins in the search for more effective vaccine candidates.
\end{abstract}

\section{Introduction}

American tegumentary leishmaniasis (ATL) is a zoonotic disease that affects man and several species of wild and domestic animals [1]. It affects around 12 million people in 88 countries, with 350 million people living in at-risk areas. It has an annual incidence of approximately 2 million new cases; of these 1.5 million cases are cutaneous leishmaniasis and 500 thousand are visceral leishmaniasis [2].

One of the strongest correlations with the polarized response in mice is the subclass profile of IgG in the antigen-specific response. Nonself-cured mice assemble an IgG1- and IgE-mediated humoral response promoted by $\mathrm{TH} 2$ cells, in contrast to mice from resistant phenotype lines that assemble a dominant humoral response and IgG2a promoted by TH1 cells. In mice, IgG2a antibodies, which are effective in complement opsonization and fixation, can act by individually targeting amastigotes released from infected macrophages [3].

Although there are chemotherapeutic agents for leishmaniasis, the drugs used are expensive, limited, toxic and threatened by drug resistance mechanisms by parasites. The prevention of leishmaniasis is superior to treatment due to the patient's health impairment and the disfigurement caused during the clinical manifestations of leishmaniasis. A prophylactic vaccination may be the most effective strategy to control the infection and spread of this group of diseases [4]. However, to date there are no reports of an effective vaccine for any form of human leishmaniasis. The fact that infected people acquire immunity against reinfections indicates the feasibility of developing a vaccine [5].

With regard to these Leishmania (L.) amazonensis membrane antigens, vaccine candidates for efficient activation of the immune system

\footnotetext{
* Corresponding author at: Fundação Oswaldo Cruz (Fiocruz Ceará), Av. Santos Dumont, 5051, sl.1303, 60175-047 Fortaleza, CE, Brazil.

E-mail address: rnicolete@gmail.com (R. Nicolete).
} 
have recently been described, including the effect of a protein-enriched membrane fraction with glycosyl-phosphatidyl inositol-GPI anchors, and proteoliposomes constructed from this fraction, in reducing the infection percentage in murine peritoneal macrophages and the percentage of intracellular amastigote cells [6]. The potential for the development of biotechnological products from micro/nanostructured antigen dispersion systems for the treatment and diagnosis of leishmaniasis was evidenced by these results. Of these protein fractions, 12 proteins were identified, requiring immunogenic characterization and identification of the other proteins present for the selection and screening of defined antigenic subunits. In this context, the purpose of this study was to develop a biodegradable polymeric micro/ nanostructured system containing membrane antigens from Leishmania and compare it to other parasite's antigens in solution.

\section{Material and methods}

\subsection{Extraction and fractionation of proteins}

Aliquots containing from 1.3 to $1.9 \times 10^{7}$ promastigotes in RPMI culture medium, subcultured for up to eight passages, were centrifuged at $1200 \times \mathrm{g}$ for $10 \mathrm{~min}$ to remove the culture medium, washed with Tris-HCl buffer ( $5 \mathrm{mM}$, pH 7.25) containing $0.2 \mathrm{mM} \mathrm{CaCl}_{2}$ (calcium chloride) and $1 \mathrm{mM} \mathrm{NaCl}$ (sodium chloride) and centrifuged again. The pellets formed were resuspended in $1 \mathrm{~mL}$ of Tris- $\mathrm{HCl}$ buffer and $5 \mu \mathrm{L}$ of a mixture of protease inhibitors (GE-Healthcare) and sonicated. The samples were frozen and stored at $-20^{\circ} \mathrm{C}$. The pellets were subjected to ultracentrifugation at $100,000 \times g$ for $1 \mathrm{~h}$ at $4^{\circ} \mathrm{C}$; the supernatant was reserved in another tube, subjected to ultrafiltration to exchange the buffer for PBS; the pellet was resuspended in PBS [6]. The fraction concentrations were measured in the presence of $2 \%$ SDS using the BCA Proten Assay kit (Thermo), according to the manufacturer's instructions.

\subsection{Production and characterization of PLGA micro/nanoparticles}

Each batch of particles was obtained by the emulsion and solvent evaporation method as described $[7,8]$, with modifications. Briefly, the stirring system and the other materials were sterilized and mounted in a laminar flow hood to ensure sterile conditions during the preparation of the particles. One hundred mg of the polymer were dissolved in each batch using PLGA 50:50 (PURASORB ${ }^{\circledR}$ 5002) in $2 \mathrm{~mL}$ of dichloromethane, sonicated for $1 \mathrm{~min}$ and thirty-six seconds at $50 \mathrm{~W}$ of power, added to a glass tube containing $9 \mathrm{~mL}$ of $1.5 \%$ polyvinyl alcohol solution, subjected for an ultrasound again for $7 \mathrm{~min}$ and $12 \mathrm{~s}$ at $50 \mathrm{~W}$ of power [6]. The resulting supernatant was discarded and the precipitate was resuspended in deionized water and centrifugation was repeated three times. The final precipitate was resuspended in $5 \mathrm{~mL}$ of deionized water for further lyophilization and storage at $-80^{\circ} \mathrm{C}$.

The distribution of the mean diameters of the micro/nanoparticulate system and the Zeta particle potential analysis was performed using a Nano Zeta Sizer laser diffractometer (Malvern instruments, England), according to the manufacturer's instructions. Briefly, $50 \mu \mathrm{L}$ of particulate suspension was dispersed in $1 \mathrm{~mL}$ of water and injected into the cuvette with a syringe for analysis.

The viability of the macrophages $\left(1 \times 10^{5}\right.$ cells/well $)$ exposed to the preparations was assessed using the MTT colorimetric method. Cells were $24 \mathrm{~h}$ incubated at $25^{\circ} \mathrm{C}$ with the micro/nanoparticles $(0.5 \mathrm{mg}$ / well). After this period, $10 \mu \mathrm{L}$ of MTT solution was added to all wells and $4 \mathrm{~h}$ later, $50 \mu \mathrm{L}$ of SDS $(20 \%, \mathrm{w} / \mathrm{v})$ was added to each well. Then, after an overnight period, the plates were gently shaken and read spectrophotometrically at $570 \mathrm{~nm}$. The cytotoxicity index was calculated using the following equation:

Cytotoxicity $=(1-$ mean OD test/mean OD of the negative control $) \times 100$.
2.3. Immunization of mice with the preparations containing antigens from $L$. amazonensis and evaluation of the response

Male BALB/C mice, 8-10 weeks old, weighing 20-28 g, obtained from the IPEPATRO/FIOCUZ-RO vivarium were used. The animals were kept under standard animal vivarium conditions. The experiments were carried out according to the rules established by the Ethical Committee on Animal Use (CEUA) at IPEPATRO-FIOCRUZ-RO, upon approval of the research project under number 2013/3.

Groups of eight male BALB/C mice were immunized subcutaneously (3 times at 3 weeks intervals) with bovine serum albumin (BSA), $L$. amazonensis soluble antigens (SLA), L. amazonensis membrane antigens (MAg), PLGA micro/nanoparticles with no protein content (P_CNT), PLGA micro/nanoparticles containing SLA (P_SLA) and PLGA nanoparticles containing MAg (P_MAg). After two weeks, mice received booster doses and one week later, they were challenged with stationary growth phase $L$. amazonensis $1 \times 10^{6}$ promastigotes. The challenge was subcutaneously applied in the right hind paw of each of the mice.

In immunization groups with non-encapsulated protein antigens, the proteins were mixed with Freund's complete adjuvant in the first dose and with Freund's incomplete adjuvant in the booster dose. In the immunization groups with the micro/nanoparticles, they were suspended in sterile saline buffer (PBS) at both doses. The volume applied was $300 \mu \mathrm{L}$, containing $40 \mu \mathrm{g}$ of particles. Two groups of eight mice each, which were not immunized, are part of the experimental controls, one in which the mice were only infected to control the challenge (INFEC) and one group in which the mice were not infected (NORMAL).

\subsection{Nitric oxide and specific antibody production in immunized mouse serum}

Half of the animals in each group were sacrificed $72 \mathrm{~h}$ after the challenge. The amount of nitric oxide produced and released in the serum of the mice was determined by the Griess assay using the procedure described [9].

Serum samples were used to determine the concentration of total IgG, IgG1 and anti-Leishmania IgG2a antibodies by enzyme-linked immunosorbent assay (ELISA) as described [10], using $40 \mu \mathrm{g} / \mathrm{ml}$ of $L$. amazonensis antigens at a ratio of 1:1000 with anti-Mouse IgG (SIGMAALDRICH M8642). Biotinylated anti-Leishmania anti-IgG1 (SIGMAALDRICH A3562), anti-IgG1 (SIGMA-ALDRICH SAB3701172) or antiIgG2a (SIGMA-ALDRICH SAB3701179), conjugated to alkaline phosphatase diluted 1:500 in 10\% PBS + FBS, were used for detection.

\subsection{Statistical analysis}

Statistical analyses of the results were performed considering the means \pm standard deviation obtained. The analysis of variance was performed by determining the significance level for $p<0.05$, applying a multiple comparison test (One-way ANOVA, followed by Tukey's posttest). GraphPad prism 5 Demo 5.03 software was used; SPSS Inc. GPW5614601-RAG-1147, 2013.

\section{Results}

The membrane $(0.54 \mathrm{mg} / \mathrm{mL})$ and soluble Leishmania proteins $(1.14 \mathrm{mg} / \mathrm{mL})$ obtained were used for the production of micro/nanoparticles. Table 1 shows PLGA control micro/nanoparticles with diameters of $512 \mathrm{~nm} \pm 264.7$ and Zeta potential of $-16 \mathrm{mV} \pm 4.21$; PLGA micro/nanoparticles containing SLAg with diameters of $637 \mathrm{~nm} \pm 177.3$ and Zeta potential of $-18.4 \mathrm{mV} \pm 5.38$; PLGA micro/nanoparticles containing MAg with diameters of $514.9 \pm 215.8 \mathrm{~nm}$ and Zeta potential with a value of $-15.3 \mathrm{mV} \pm$ 4.14 .

All preparations containing Leishmania antigens presented low toxicity on murine macrophages. Particles containing SLA and MAg 
Table 1

Results of particles' size, Zeta potential and \% of cytotoxicity.

\begin{tabular}{llll}
\hline Formulations & Size $(\mathrm{nm})$ & Zeta potential $(\mathrm{mV})$ & Cytotoxicity (\%) \\
\hline P_CNT & $512 \pm 264$ & $-16.0 \pm 4.21$ & n.d. \\
P_SLA & $637 \pm 167.3$ & $-18.4 \pm 5.38$ & 4.35 \\
P_MAg & $514.9 \pm 214.8$ & $-18.4 \pm 5.38$ & 19.22 \\
\hline
\end{tabular}

Size (nm), Zeta potential (mV) and cytotoxicity assay of control particles (P_CNT), those containing soluble antigens (P_SLA) and those containing membrane antigens from $L$. amazonensis (P_MAg). The values were obtained from two different batches of particles conducted in triplicate.

n.d. $=$ not detected.

showed $4.3 \%$ and $19.2 \%$ of cytotoxicity, respectively (Table 1 ).

Experimental groups composed of animals that were immunized with non-encapsulated protein antigens showed higher NO serum production compared to encapsulated ones after the challenge with $L$. amazonensis. MAg caused the highest NO production (about $80 \mu \mathrm{M}$ ). Of the micro/nanoencapsulated formulations, P_MAg showed the highest level (about $20 \mu \mathrm{M}$ ) (Fig. 1A).

The sera of animals immunized with the non-encapsulated antigen formulations (SLA and MAg) showed higher intensity of total IgGs compared to all other immunized groups (Fig. 1B). Interestingly, although lower levels of IgGs were detected in the sera of the mice that were immunized with the micro/nanoparticulated preparations, the IgG2a/IGg1 ratio was $2 \times$ higher for P_SLA (1) compared to SLA (0.5) and $10 \times$ higher for P_MAg (2) compared to MAg (0.2) immunized group.

\section{Discussion}

The parasites were grouped by passages in order to make extracts with homogeneous protein composition representative of infective promastigotes, ensuring that the material used in immunizations with and without adjuvants and/or micro/nanostructured antigen dispersion systems are defined and reproducible as to the ability for immune response modulation, since the successive transfer in culture can cause a change in the virulence of parasites of the genus Leishmania [11].

The polymeric particles' production method employed allowed for the production of micro/nanoparticles with desired diameter and Zeta potential characteristics for a vaccinal approach. Microscopic particles can establish a long-term antigen release profile, enhancing the exposure of the antigens to antigen-presenting cells in the animals and consequently the induction of immune memory after challenge with the parasite [12].

The procedure for particle production was reassessed and standar-

A

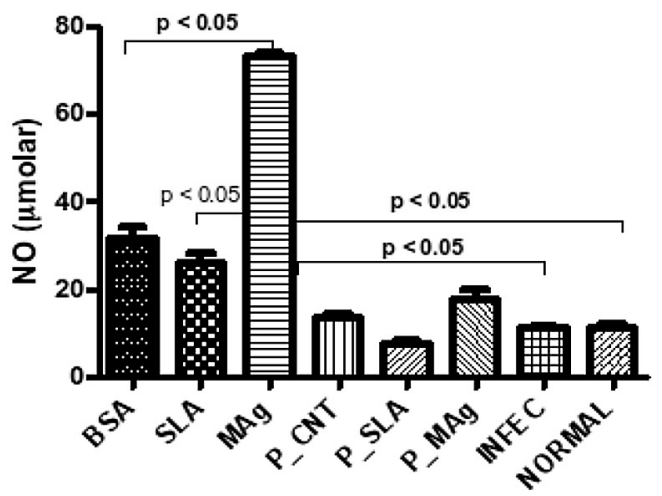

dized to obtain batches of particles with diameters from $512 \mathrm{~nm}$ to $637 \mathrm{~nm}$. Particles smaller than $1 \mathrm{~mm}$ may have a larger surface area, influencing the release rate of the antigens. Microparticles induce polarization of the immune response to the TH2 pathway and nanoparticles to the TH1 pathway [13].

In this study, we also speculated about three components of signaling pathways, especially proinflammatory ones, which were mostly activated during the macrophages' incubation $(24 \mathrm{~h})$ with the micro/nanoparticulate preparations. The encapsulated membrane antigen formulation (P_MAg) was able to increase 1.46, 1.16 and 1.03 (in arbitrary units) for NF- $\mathrm{BB}$ p65, phospho p38 and STAT3 signaling pathways, respectively. These results are also consistent with the cytokine profile produced by the stimuli with P_MAg, showing high levels of TNF- $\alpha(500 \mathrm{pg} / \mathrm{mL})$ and IL-6 $(130 \mathrm{pg} / \mathrm{mL})$ in the cell supernatants.

According to these results, it is known that activation of STAT3 signaling pathway would indicate a reaction of inflammatory processes, such as lymphocyte proliferation and maturation and inflammation regulation, being activated by IL-6 [14], corroborating the suggestion that micro/nanostructured preparations, especially that containing MAg, was able to stimulate macrophages at the molecular level for the production of an inflammatory response.

The protection or progression of the disease in murine ATL models is related to the TH1 and TH2 immune response profiles, respectively, which can be characterized by the cytokine profile in each pathway, markedly IL-12, IFN- $\gamma$ and TNF- $\alpha$ in the TH1; IL- 4 and IL- 6 pathways in the TH2 pathway [15], as well as the production of chemokines responsible for the polarization of $\mathrm{T}$ helper cells and the coordination mechanisms of nitric oxide production in macrophages [16]. IFN- $\gamma$ induces the isotype exchange from IgM to IgG2a, whereas IL-4 promotes the exchange of IgM isotypes for IgG1 and IgE [17]. Infection control as a function of a TH1 response occurs through the elimination of parasites in macrophages by microbicidal mechanisms mediated by reactive oxygen species, such as nitric oxide (NO). IFN- $\gamma$ and TNF- $\alpha$ act synergistically in the induction of NO production in macrophages $[18,19]$.

The results obtained from the immunization experiments revealed that animals immunized with the free or encapsulated membrane fraction (MAg) were able to produce high amounts of NO. However, encapsulated parasite fractions proved to be better inducers of a specific TH1 response (IGg2a) as opposed to free ones. More studies will be conducted in order to clarify these opposite behaviors of the parasites' antigens and the role of ach formulation in stimulating the host cellular and/or humoral immune response.

B

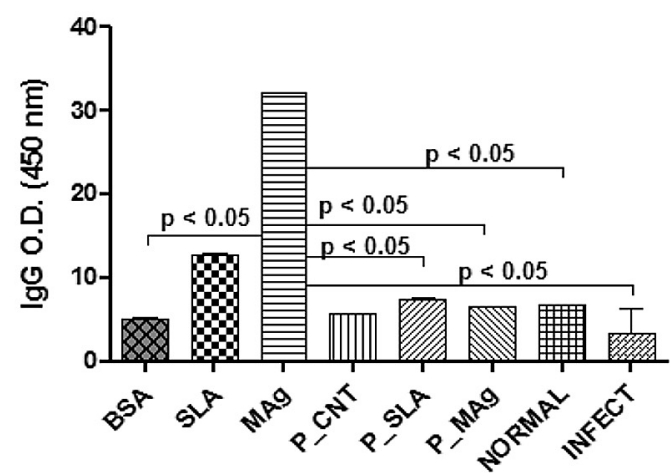

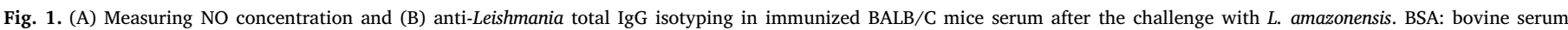

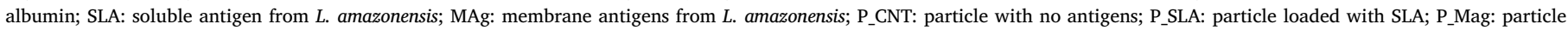

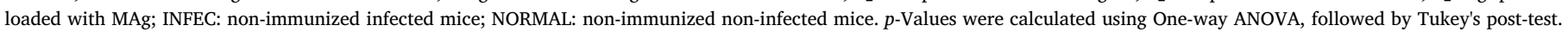
Two set of experiments were conducted. 


\section{Acknowledgements}

The authors express their gratitude to Johnny Ramos from the Laboratory of Immunophysiology (UFMA) for his technical contribution in CBA experiments. Fundação Oswaldo Cruz (FIOCRUZ), Conselho Nacional de Desenvolvimento Científico e Tecnológico ( $\mathrm{CNPq}, \mathrm{n}$. 470455/2013-6) and Coordenação de Aperfeiçoamento de Pessoal de Nível Superior (CAPES) were responsible for financial support.

\section{References}

[1] Brasil. Ministério da Saúde, Manual de Vigilândia em Leishmaniose Tegumentar americana, second ed., Ministério da Saúde, Brasília, DF, 2007.

[2] P. Mitropoulos, P. Konidas, M. Durkin-Konidas, New World cutaneous leishmaniasis: updated review of current and future diagnosis and treatment, J. Am. Acad. Dermatol. 63 (2010) 309-322, http://dx.doi.org/10.1016/j.jaad.2009.06.088.

[3] M.J. Day, Immunoglobulin G subclass distribution in canine leishmaniosis: a review and analysis of pitfalls in interpretation, Vet. Parasitol. 147 (2007) 2-8, http://dx. doi.org/10.1016/j.vetpar.2007.03.037.

[4] N. Dunning, Leishmania vaccines: from leishmanization to the era of DNA technology, Biosci. Horiz. 2 (2009) 73-82, http://dx.doi.org/10.1093/biohorizons/ hzp004.

[5] R. Nagill, S. Kaur, Vaccine candidates for leishmaniasis: a review, Int. Immunopharmacol. 11 (2011) 1464-1488, http://dx.doi.org/10.1016/j.intimp. 2011.05.008.

[6] M.C. Colhone, I. Silva-Jardim, R.G. Stabeli, P. Ciancaglini, Nanobiotechnologic approach to a promising vaccine prototype for immunisation against leishmaniasis: a fast and effective method to incorporate GPI-anchored proteins of Leishmania amazonensis into liposomes, J. Microencapsul. 32 (2015) 143-150, http://dx.doi. org/10.3109/02652048.2014.958203.

[7] T. Niwa, H. Takeuchi, T. Hino, N. Kunou, Y. Kawashima, Preparations of biodegradable nanospheres of water-soluble and insoluble drugs with D,L-lactide/ glycolide copolymer by a novel spontaneous emulsification solvent diffusion method, and the drug release behavior, J. Control. Release 25 (1993) 89-98, http:// dx.doi.org/10.1016/0168-3659(93)90097-O.

[8] R. Nicolete, K.M. Lima, J.M.R. Júnior, M.D. Baruffi, A.I. de Medeiros, M.V.L.B. Bentley, C.L. Silva, L.H. Faccioli, In vitro and in vivo activities of leukotriene B4-loaded biodegradable microspheres, Prostaglandins Other Lipid Mediat. 83 (2007) 121-129, http://dx.doi.org/10.1016/j.prostaglandins.2006.10.
007.

[9] N.B. De Barros, S.R.A. Macedo, A.S. Ferreira, M.P. Tagliari, F.B. Zanchi, A.M. Kayano, A.M. Soares, R. Nicolete, Liposomes containing an ASP49-phospholipase A2 from Bothrops jararacussu snake venom as experimental therapy against cutaneous leishmaniasis, Int. Immunopharmacol. 36 (2016) 225-231, http://dx. doi.org/10.1016/j.intimp.2016.04.025.

[10] R. Thorpe, A. Johnstone, Immunoassays, in: A. Johnstone, R. Thorpe (Eds.), Immunochemistry in Practice, second ed., Blackwell Scientific Publications, London, 1987, pp. 241-260.

[11] B. Espiau, V. Vilhena, A. Cuvillier, A. Barral, G. Merlin, Phenotypic diversity and selection maintain Leishmania amazonensis infectivity in BALB/C mouse model, Mem Inst Oswaldo Cruz Rio Janeiro 112 (2017) 44-52, http://dx.doi.org/10.1590/ 0074-02760160280.

[12] S. Gordon, J.C. Unkeless, Z.A. Cohn, Induction of macrophage plasminogen activator by endotoxin stimulation and phagocytosis, J. Exp. Med. 140 (1974) 995-1010.

[13] I. Gutierro, R.M. Hernández, M. Igartua, A.R. Gascón, J.L. Pedraz, Size dependent immune response after subcutaneous, oral and intranasal administration of BSA loaded nanospheres, Vaccine 21 (2002) 67-77, http://dx.doi.org/10.1016/S0264410X(02)00435-8.

[14] T. ZARUBIN, J. HAN, Activation and signaling of the p38 MAP kinase pathway, Cell Res. 15 (2005) 11-18, http://dx.doi.org/10.1038/sj.cr.7290257.

[15] J. Ji, J. Sun, L. Soong, Impaired expression of inflammatory cytokines and chemokines at early stages of infection with Leishmania amazonensis, Infect. Immun. 71 (2003) 4278-4288, http://dx.doi.org/10.1128/iai.71.8.4278-4288.2003.

[16] S. Bhattacharyya, S. Ghosh, B. Dasgupta, D. Mazumder, S. Roy, S. Majumdar, Chemokine induced leishmanicidal activity in murine macrophages via the generation of nitric oxide, J. Infect. Dis. 185 (2002) 1704-1708, http://dx.doi.org/ $10.1086 / 340820$

[17] M.R. Mohammadi, M. Zeinali, S.K. Ardestani, A. Kariminia, Identification of novel Leishmania major antigens that elicit IgG2a response in resistant and susceptible mice, Korean J. Parasitol. 44 (2006) 43-48, http://dx.doi.org/10.3347/kjp.2006. 44.1.43.

[18] F.Y. Liew, S. Millott, C. Parkinson, R.M. Palmer, S. Moncada, Macrophage killing of Leishmania parasite in vivo is mediated by nitric oxide from L-arginine, J. Immunol. 144 (1990).

[19] D.M. Santos, M.W. Carneiro, T.R. de Moura, K. Fukutani, J. Clarencio, M. Soto, S. Espuelas, C. Brodskyn, A. Barral, M. Barral-Netto, C.I. de Oliveira, Towards development of novel immunization strategies against leishmaniasis using PLGA nanoparticles loaded with kinetoplastid membrane protein-11, Int. J. Nanomedicine 7 (2012) 2115-2127, http://dx.doi.org/10.2147/IJN.S30093. 